\title{
Integral points on the homogeneous cone
}

$$
z^{2}=3 x^{2}+6 y^{2}
$$

\author{
M. A. Gopalan ${ }^{1}$, B. Sivakami ${ }^{2}$ \\ ${ }^{1}$ (Department of Mathematics ,Shrimati Indira Gandhi College, Tiruchirapalli-620002,Tamilnadu,India) \\ ${ }^{2}$ (Department of Mathematics, Chettinad College of Engineering and Technology, Karur-639114,
}

Tamilnadu,India)

\begin{abstract}
The homogeneous cone represented by the ternary quadratic equation $z^{2}=3 x^{2}+6 y^{2}$ is analysed for its non-zero integral solutions. Five different patterns of solutions are illustrated. In each pattern, interesting relations among the solutions and some special polygonal and pyramidal numbers are exhibited.

Keywords: Homogeneous cone, Integral solutions, Polygonal numbers, Pyramidal numbers, Ternary quadratic.

\section{Introduction}

The ternary quadratic Diophantine equations offer an unlimited field for research by reason of variety[1-2]. For an extensive review of various problems one may search refer [3-15]. This communication concerns with yet another interesting ternary quadratic equation representing a homogeneous cone for determining its infinitely many non-zero integral solutions.Also a few interesting relations among the solutions and some special polygonal and pyramidal numbers are presented. Further three different general forms for generating sequence of integral points based on the given point on the considered cone are exhibited.
\end{abstract}

\section{Notations}

\begin{tabular}{|l|l|l|}
\hline Polygonal Numbers & Notations for rank 'n' & Definitions \\
\hline Triangular number & $\mathrm{T}_{\mathrm{n}}$ & $\frac{1}{2} \mathrm{n}(\mathrm{n}+1)$ \\
\hline Pentagonal number & $\mathrm{Pen}_{\mathrm{n}}$ & $\frac{1}{2}\left(3 \mathrm{n}^{2}-\mathrm{n}\right)$ \\
\hline Hexagonal number & $\mathrm{Hex}_{\mathrm{n}}$ & $2 \mathrm{n}^{2}-\mathrm{n}$ \\
\hline Octagonal number & $\mathrm{Oct}_{\mathrm{n}}$ & $3 \mathrm{n}^{2}-2 \mathrm{n}$ \\
\hline Nanogonal number & $\mathrm{Nan}_{\mathrm{n}}$ & $\frac{1}{2}\left(7 \mathrm{n}^{2}-5 \mathrm{n}\right)$ \\
\hline Decagonal number & $\mathrm{Dec}_{\mathrm{n}}$ & $4 \mathrm{n}^{2}-3 \mathrm{n}$ \\
\hline Hendecagonal number & $\mathrm{HD}_{\mathrm{n}}$ & $\frac{1}{2}\left(9 \mathrm{n}^{2}-7 \mathrm{n}\right)$ \\
\hline Dodecagonal number & $\mathrm{DD}_{\mathrm{n}}$ & $\frac{1}{2}\left(10 \mathrm{n}^{2}-8 \mathrm{n}\right)$ \\
\hline Tridecagonal number & $\mathrm{TD}_{\mathrm{n}}$ & $\frac{1}{2}\left(11 \mathrm{n}^{2}-9 \mathrm{n}\right)$ \\
\hline Tetradecagonal number & $\mathrm{TED}_{\mathrm{n}}$ & $\frac{1}{2}\left(12 \mathrm{n}^{2}-10 \mathrm{n}\right)$ \\
\hline Octadecagonal number & $\mathrm{OD}_{\mathrm{n}}$ & $\frac{1}{2}\left(16 \mathrm{n}^{2}-14 \mathrm{n}\right)$ \\
\hline Icosagonal number & $\mathrm{IC}_{\mathrm{n}}$ & $\frac{1}{2}\left(18 \mathrm{n}^{2}-16 \mathrm{n}\right)$ \\
\hline Centered Square number & $\mathrm{CS}_{\mathrm{n}}$ & $\mathrm{n}^{2}+(\mathrm{n}-1)^{2}$ \\
\hline & & \\
\hline
\end{tabular}


Integral points on the homogeneous cone $z^{2}=3 x^{2}+6 y^{2}$

\begin{tabular}{|l|l|l|}
\hline Centered hexagonal number & $\mathrm{CH}_{n}$ & $3 \mathrm{n}^{2}-3 \mathrm{n}+1$ \\
\hline Gnomonic number & $\mathrm{Gno}_{\mathrm{n}}$ & $2 \mathrm{n}-1$ \\
\hline Pronic number & $\mathrm{Pro}_{\mathrm{n}}$ & $\mathrm{n}(\mathrm{n}+1)$ \\
\hline Stella Octangula number & $\mathrm{SO}_{\mathrm{n}}$ & $\mathrm{n}\left(2 \mathrm{n}^{2}-1\right)$ \\
\hline Star number & $\mathrm{Star}_{\mathrm{n}}$ & $6 \mathrm{n}(\mathrm{n}-1)+1$ \\
\hline Pentagonal Pyramidal number & $\mathrm{PP}_{\mathrm{n}}$ & $\frac{1}{2} \mathrm{n}^{2}(\mathrm{n}+1)$ \\
\hline Hexagonal Pyramidal number & $\mathrm{HXP}_{\mathrm{n}}$ & $\frac{1}{6} \mathrm{n}(\mathrm{n}+1)(4 \mathrm{n}-1)$ \\
\hline Tetrahedral number & $\mathrm{Tetra}_{\mathrm{n}}$ & $\frac{1}{6} \mathrm{n}(\mathrm{n}+1)(\mathrm{n}+2)$ \\
\hline Pentatope number & $\mathrm{PT}_{\mathrm{n}}$ & $\frac{1}{24} \mathrm{n}(\mathrm{n}+1)(\mathrm{n}+2)(\mathrm{n}+3)$ \\
\hline
\end{tabular}

\section{Method Of Analysis}

The equation under consideration to be solved is $z^{2}=3 x^{2}+6 y^{2}$

Five different patterns to (1) are illustrated below:

\section{Pattern 1:}

By applying the transformations, $x=X+6 T, y=X-3 T, z=3 W$

equation (1) reduces to $W^{2}=X^{2}+18 T^{2}$

which is satisfied by $T=2 r s, X=18 r^{2}-s^{2}, W=18 r^{2}+s^{2}$

Thus in view of (2), the non-zero distinct integral points on the homogeneous cone (1) are given by

$$
\begin{aligned}
& x(r, s)=18 r^{2}+12 r s-s^{2} \\
& y(r, s)=18 r^{2}-6 r s-s^{2} \\
& z(r, s)=54 r^{2}+3 s^{2}
\end{aligned}
$$

\subsection{Properties:}

1. Each of the following represents a Nasty number:
(i) $x(r, 1)+2 y(r, 1)+3$
(ii) $z(r, 1)-3$
(iii) $z(r, s)-x(r, s)-2 y(r, s)$

2. Each one of the following is a perfect square:

(i) $3 x(r, s)+6 y(r, s)+3 z(r, s)$

(ii) $12 x(1, s)+6 y(1, s)+3$ star $_{S}-120 T_{S}+30$ Hex $_{S}-3$

3. $3 x(r, 1)-z(r, 1)-18 G$ noo $_{r} \equiv 0(\bmod 12)$

4. $x(1, s)+\operatorname{Dec}_{S}-O c t_{S}-18 \equiv 0(\bmod 11)$

5. $y(1, s)+\operatorname{Pr} o_{S}-18 \equiv 0(\bmod 5)$

6. $x(r, 1)-36 T_{r}+3 G n o_{r}+4=0$

7. $y(r, 1)-6\left[T E D_{r}-D D_{r}+C S_{r}+G n o_{r}\right]+1=0$

8. $2 z(1, s)-3\left[T E D_{S}-D D_{S}+\operatorname{Pr} o_{S}\right]-108=0$

\section{Pattern 2:}

Consider (3) as $X^{2}+18 T^{2}=W^{2} * 1$ 
Let $W=a^{2}+18 b^{2}$ and write 1 as $1=\frac{(1+i 2 \sqrt{2})(1-i 2 \sqrt{2})}{9}$

Then $(4) \Rightarrow(X+i 3 \sqrt{2} T)(X-i 3 \sqrt{2} T)=(a+i 3 \sqrt{2} b)^{2}(a-i 3 \sqrt{2} b)^{2} \frac{(1+i 2 \sqrt{2})(1-i 2 \sqrt{2})}{9}$

Let us define $X+i 3 \sqrt{2} T=(a+i 3 \sqrt{2} b)^{2} \frac{(1+i 2 \sqrt{2})}{3}$

By equating the real and imaginary parts on both sides, we get

$$
\begin{aligned}
X & =\frac{1}{3}\left(a^{2}-24 a b-18 b^{2}\right) \\
T & =\frac{1}{9}\left(2 a^{2}+6 a b-36 b^{2}\right)
\end{aligned}
$$

Using these values of $\mathrm{X}$ and $\mathrm{T}$ the values of $\mathrm{x}, \mathrm{y}$ and $\mathrm{z}$ are obtained as

$$
\begin{aligned}
& x=\frac{1}{3}\left(5 a^{2}-12 a b-90 b^{2}\right) \\
& y=\frac{1}{3}\left(-a^{2}-30 a b+18 b^{2}\right) \\
& z=3 a^{2}+54 b^{2}
\end{aligned}
$$

Since our aim is to get integral values, we may choose $a=3 \mathrm{~A}$ and $\mathrm{b}=3 \mathrm{~B}$

Then the solutions of (1) are given by

$$
\begin{aligned}
& x(A, B)=15 A^{2}-36 A B-270 B^{2} \\
& y(A, B)=-3 A^{2}-90 A B+54 B^{2} \\
& z(A, B)=27 A^{2}+486 B^{2}
\end{aligned}
$$

\subsection{Properties:}

1. Each of the following represents a perfect square:

(i) $3[x(A, A)+z(A, A)]+15 y(A, A)$

(ii) $-3 y(A, 1)-135$ Gno $_{A}+27$

2. $y(A, 1)+z(A, 1)-x(A, 1)-3 \operatorname{Oct}_{A}+24$ Gno $_{A}-786=0$

3. $z(A, 1)-y(A, 1)-60 \operatorname{Pr} o_{A}+15 C S_{A} \equiv 0(\bmod 477)$

4. $x(A, 1)+5 y(A, 1)=54 D D_{A}-540 T_{A}$

5. Each one of the following represents a Nasty number:

(i) $x(A, 1)+z(A, 1)-12 \mathrm{Nan}_{A}+12 \mathrm{Hex}_{A}-6 \operatorname{Dec}_{A}$

(ii) $2 z(A, 1)-972$

6. $x(A, 1)-6\left(O D_{A}\right)+6 T D_{A}+42 H D_{A}-21 I C_{A} \equiv 0(\bmod 270)$

7. $5 D D_{z}-4 T E D_{z}=2 T_{x}+$ Hex $_{x}+18$ Oct $_{y}-12 D e c_{y}$

8. $\left(\frac{P P_{z}}{T_{z}}\right)^{2}=3\left(\frac{4 P T_{x}}{\text { Tetra }_{x}}-3\right)^{2}+6\left(\frac{6 \text { Tetra }}{\operatorname{Pr} o_{y}}-2\right)^{2}$

Taking the transformations $x=2 u+1, y=u-1, z=3 v$

equation (1) is reduced to $v^{2}=2 u^{2}+1$

whose general solution is $v_{S}=\frac{f}{2}, u_{S}=\frac{g}{2 \sqrt{2}}$

Here $f=(3+2 \sqrt{2})^{s+1}+(3-2 \sqrt{2})^{s+1}$ 


$$
\mathrm{g}=(3+2 \sqrt{2})^{s+1}-(3-2 \sqrt{2})^{s+1}
$$

From these values of $v_{S}, u_{S}$, the solutions of (1) are given by

$$
x_{S}=\frac{g}{\sqrt{2}}+1, y_{S}=\frac{g}{2 \sqrt{2}}-1, z_{S}=\frac{3 f}{2}
$$

The recurrence relations for the solutions are

$$
\begin{aligned}
& x_{S+2}-6 x_{S+1}+x_{S}=-4 \\
& y_{S+2}-6 y_{S+1}+y_{S}=4 \\
& z_{S+2}-6 z_{S+1}+z_{S}=0 \quad \mathrm{~s}=0,1,2,3, \ldots
\end{aligned}
$$

Using the above recurrence relations, few integral solutions for (1) are presented as follows:

\begin{tabular}{|c|c|c|c|}
\hline $\mathbf{s}$ & $\mathbf{x}_{\mathbf{s}}$ & $\mathbf{y}_{\mathbf{s}}$ & $\mathbf{z}_{\mathbf{s}}$ \\
\hline 0 & 5 & 1 & 9 \\
\hline 1 & 25 & 11 & 51 \\
\hline 2 & 141 & 69 & 297 \\
\hline 3 & 817 & 407 & 1731 \\
\hline 4 & 4757 & 2377 & 10089 \\
\hline 5 & 27721 & 13859 & 58803 \\
\hline 6 & 161565 & 80781 & 342729 \\
\hline
\end{tabular}

\subsection{Properties:}

1. $x_{s}-2 y_{s} \equiv 0(\bmod 3)$

2. $x_{s+2}-12 y_{s+1}+x_{s} \equiv 0(\bmod 14)$

3. $63 x_{S+1}-378 y_{S}-84 z_{S}$ is a perfect square.

4. $6 z_{s+2}-216 x_{S}-102 z_{S}+216=0$

5. $2 z_{S+1}-6 z_{S}-24 y_{S}$ is a Nasty number.

6. $2 z_{s+1}-4 x_{s+1}-y_{s+1}+3 y_{s} \equiv 0(\bmod 6)$

7. $\left(x_{S}-1\right)\left(y_{S}+1\right)$ is a perfect square.

\section{Pattern 4:}

Equation (1) can be rewritten as $z^{2}-6 y^{2}=3 x^{2}$

Let $x=a^{2}-6 b^{2}$ and $3=(3+\sqrt{6})(3-\sqrt{6})$

Then $(6) \Rightarrow(z+\sqrt{6} y)(z-\sqrt{6} y)=(3+\sqrt{6})(3-\sqrt{6})\left[(a+\sqrt{6} b(a-\sqrt{6})]^{2}\right.$

Define $z+\sqrt{6} y=(3+\sqrt{6})(a+\sqrt{6} b)^{2}$

By equating the real and imaginary parts, the values of $y$ and $z$ are

$$
\begin{aligned}
& y=a^{2}+6 a b+6 b^{2} \\
& z=3 a^{2}+12 a b+18 b^{2}
\end{aligned}
$$

Then the solutions of (1) are represented by $x(a, b)=a^{2}-6 b^{2}$

$$
\begin{aligned}
& y(a, b)=a^{2}+6 a b+6 b^{2} \\
& z(a, b)=3 a^{2}+12 a b+18 b^{2}
\end{aligned}
$$

\subsection{Properties:}

1. Each of the following is a perfect square:

(i) $x(a, 1)+6$ is a perfect square.

(ii) $z(a, 1)-3 x(a, 1)-6 \operatorname{Pr} o_{a}+3 C S_{a}-3$

2. $y(a, 1)-2 T_{a}-6 \equiv 0(\bmod 5)$ 
3. $2 z(a, 1)-3 \operatorname{Pr} o_{a}-\mathrm{CH}_{a}-12 \mathrm{Gno}_{a}-47=0$

4. $z(a, 1)-2 y(a, 1)-x(a, 1) \equiv 0(\bmod 12)$

5. $4 x(a, 1)-\frac{3 H X P a^{2}}{T_{a}}+23=0$

6. $6 z(a, 1)-12 y(a, 1)-36$ is a Nasty number.

7. $7 x(a, 1)-y(a, 1)-$ Star $_{a}+49=0$

8. $6 x(a, 1)-\mathrm{CH}_{a}-6 T_{a} \equiv 0(\bmod 37)$

\section{Pattern 5:}

Equation (1) can also be written as $z^{2}-3 x^{2}=6 y^{2}$

Let $y=a^{2}-3 b^{2}$ and $6=(3+\sqrt{3})(3-\sqrt{3})$

From (7), $(z+\sqrt{3} x)(z-\sqrt{3} x)=(3+\sqrt{3})(3-\sqrt{3})[(a+\sqrt{3} b)(a-\sqrt{3} b)]^{2}$

Let us define $z+\sqrt{3} x=(3+\sqrt{3})(a+\sqrt{3} b)^{2}$

By Equating real and imaginary parts, we get

$$
\begin{aligned}
& x=a^{2}+6 a b+3 b^{2} \\
& z=3 a^{2}+6 a b+9 b^{2}
\end{aligned}
$$

Then the solutions of (1) are $x(a, b)=a^{2}+6 a b+3 b^{2}$

$$
\begin{aligned}
& y(a, b)=a^{2}-3 b^{2} \\
& z(a, b)=3 a^{2}+6 a b+9 b^{2}
\end{aligned}
$$

\subsection{Properties:}

1. $z(a, 1)-y(a, 1)-x(a, 1)$ can be written as the sum of two perfect squares.

2. Each of the following is a perfect square

(i) $2[x(a, 1)+y(a, 1)]-24 \operatorname{Pr} o_{a}+48 P P_{a}-12 S_{a}$

(ii) $z(1, b)-18 \mathrm{Hex}_{b}+12 \mathrm{Oct}_{b}-3$

3. $6 z(a, 1)-60 T_{a}+6 H_{e x}$ is a Nasty number.

4. $x(a, 1)+y(a, 1)-C S_{a}-4 G n o_{a} \equiv 0(\bmod 3)$

5. $z(a, 1)-x(a, 1)-2 C S_{a}+C H_{a}-\operatorname{Pr} o_{a} \equiv 0(\bmod 5)$

6. $2 y(1, b)+3$ Pen $_{b}+3 T_{b}=2$

7. $4 x(1, b)-3 C S_{b}-6 \operatorname{Pr} o_{b}-12 G n o_{b} \equiv 0(\bmod 13)$

\section{Remarkable observations:} satisfies (1).

If $\left(x_{0}, y_{0}, z_{0}\right)$ is any given solution of (1), then each of the following three triples (i to iii) of integers

(i) $\left(x_{n}, y_{0}, z_{n}\right)$, where $x_{n}=Y_{n-1} x_{0}+X_{n-1} z_{0}, z_{n}=3 X_{n-1} x_{0}+Y_{n-1} z_{0}, \mathrm{n}=1,2,3, \ldots$

$\left[\left(X_{n-1}, Y_{n-1}\right)\right.$ is the general solution of $\left.Y^{2}=3 X^{2}+1\right]$

(ii) $\left(x_{0}, y_{n}, z_{n}\right)$, where $y_{n}=Y_{n-1} y_{0}+X_{n-1} z_{0}, z_{n}=6 X_{n-1} y_{0}+Y_{n-1} z_{0}, \mathrm{n}=1,2,3, \ldots$

$\left[\left(X_{n-1}, Y_{n-1}\right)\right.$ is the general solution of $\left.Y^{2}=6 X^{2}+1\right]$

(iii) $\left(x_{n}, y_{n}, z_{n}\right)$, where $x_{n}=\frac{1}{3}\left\{\left[3^{n}+2(-3)^{n}\right] x_{0}+\left[2.3^{n}-2(-3)^{n}\right] y_{0}\right\}$,

$$
y_{n}=\frac{1}{3}\left\{\left[3^{n}-(-3)^{n}\right] x_{0}+\left[2.3^{n}+(-3)^{n}\right] y_{0}\right\} \text { and } z_{n}=3^{n} z_{0}, \mathrm{n}=1,2,3, \ldots
$$




\section{Conclusion}

One may search for other patterns of solutions and relations among the solutions, and also the relations between the solutions and polygonal numbers.

\section{References}

[1] L.E.Dickson, History of theory of numbers, Vol 2, Chelsea publishing company, New York, 1952

[2] Mordell, L.J.,Diophantine Equations, Academic Press, London,1969.

[3] M.A.Gopalan and D.Geetha, Lattice points on the hyperboloid of one sheet $x^{2}-6 x y+y^{2}+6 x-2 y+5=z^{2}+4$, Impact J.Sci.Tech. Vol..4, No. 1, 2010,23-32.

[4] M.A.Gopalan and V.Pandichelvi, Integral solutions of Ternary quadratic Equation $Z(X-Y)=4 X Y$, Impact J.Sci.Tech., Vol. 5(1),2011, pp. 1-6.

[5] M.A.Gopalan and J.Kaliga Rani, On Ternary Quadratic Equation $X^{2}+Y^{2}=Z^{2}+8$, Impact J.Sci.Tech., Vol. 5(1), 2011, pp. 3943.

[6] M.A.Gopalan, S.Vidhyalakshmi and T.R.Usharani, Integral points on the homogeneous cone $2 z^{2}+4 x y+8 x-4 z+2=0$, Global Journal of Mathematics and Mathematical Sciences, Vol. 2(1), 2012, pp. 61-67.

[7] M.A.Gopalan, S.Vidhyalakshmi, T.R.Usharani and S.Mallika, Integral points on the Homogeneous cone $6 z^{2}+3 y^{2}-2 x^{2}=0$, Impact J.Sci.Tech. Vol.6(1), 2012,pp. 7-13.

[8] M.A.Gopalan, S.Vidhyalakshmi and K.Lakshmi, Integral points on the hyperboloid of two sheets $3 y^{2}=7 x^{2}-z^{2}+21$, Diophantus J. Math., 1(2), 2012, 99-107.

[9] M.A.Gopalan, S.Vidhyalakshmi and E.Premalatha, On Ternary Quadratic Diophantine Equation $x^{2}+3 y^{2}=7 z^{2}$, Diophantus $J$. Math., 1(1), 2012, 51-57

[10] M.A.Gopalan, S.Vidhyalakshmi and A.Kavitha, Integral points on the homogeneous cone $z^{2}=2 x^{2}-7 y^{2}$, Diophantus J. Math., 1(2), 2012, 127-136.

[11] M.A.Gopalan, S.Vidhyalakshmi and S.Mallika, Observations on Hyperboloid on one sheet $x^{2}+2 y^{2}-z^{2}=2$, Bessel J.Math,2(3), 2012, 221-226.

[12] M.A.Gopalan, S.Vidhyalakshmi and G.Sumathi, Lattice points on the Elliptic paraboloid $9 x^{2}+4 y^{2}=z$, Advances in Theoretical and Applied Mathematics, Vol. 7, 2012, pp. 379-385.

[13] M.A.Gopalan, V.Sangeetha and Manju Somnath, On the Ternary Quadratic Equation $5\left(x^{2}+y^{2}\right)-9 x y=19 z^{2}$, International Journal of Innovative Research in Science, Engineering and Technology, Vol. 2, Issue 6, June 2013.

[14] M.A.Gopalan and V.Geetha, Lattice points on the homogeneous cone $z^{2}=2 x^{2}+8 y^{2}-6 x y$, Indian Journal of Science, Vol. 2, No. 4, Feb 2013.

[15] S.Vidhyalakshmi, M.A.Gopalan and A.Kavitha, Observations on the hyperboloid of two sheets $7 X^{2}-3 Y^{2}=Z^{2}+Z(Y-X)+4$, International Journal of Latest Research in Science, Engineering and Technology, Vol. 2, Issue 2, March-April 2013, Pg. No. 8486.

[16] S.Vidhyalakshmi, K.Lakshmi and M.A.Gopalan, Lattice points on the Elliptic paraboloid $16 y^{2}+9 z^{2}=4 x$, Bessel J. Math., 3(2),2013, 137-145.

[17] M.A.Gopalan, V.Sangeetha and Manju Somnath, Integral points on the homogeneous cone $z^{2}=5 x^{2}+11 y^{2}$, Discovery Science, Vol. 3, 2013, pp. 5-8. 